\title{
Quasi-bound state resonances of charged massive scalar fields in the near-extremal Reissner-Nordström black-hole spacetime
}

\author{
Shahar Hod ${ }^{1,2, a}$ \\ ${ }^{1}$ The Ruppin Academic Center, Emeq Hefer 40250, Israel \\ 2 The Hadassah Academic College, Jerusalem 91010, Israel
}

Received: 29 March 2017 / Accepted: 12 May 2017 / Published online: 26 May 2017

(C) The Author(s) 2017. This article is an open access publication

\begin{abstract}
The quasi-bound states of charged massive scalar fields in the near-extremal charged Reissner-Nordström black-hole spacetime are studied analytically. These discrete resonant modes of the composed black-hole-field system are characterized by the physically motivated boundary condition of ingoing waves at the black-hole horizon and exponentially decaying (bounded) radial eigenfunctions at spatial infinity. Solving the Klein-Gordon wave equation for the linearized scalar fields in the black-hole spacetime, we derive a remarkably compact analytical formula for the complex frequency spectrum which characterizes the quasi-bound state resonances of the composed ReissnerNordström-black-hole-charged-massive-scalar-field system.
\end{abstract}

\section{Introduction}

The powerful no-hair theorems of Bekenstein and Mayo [1] (see also [2-34] $)^{1}$ have revealed the fact that, in asymptotically flat spacetimes, spherically symmetric charged black holes cannot support external static matter configurations made of charged massive scalar fields. It should be emphasized, however, that these mathematically elegant no-hair theorems [1] do not address the following physically interesting question: how long does it take for a newly born charged black hole to shed (that is, to swallow or to radiate to infinity) the external charged fields?

Interestingly, recent numerical investigations $[35,36]$ of the Klein-Gordon wave equation for charged massive scalar fields in the spherically symmetric Reissner-Nordström

\footnotetext{
${ }^{1}$ It is worth mentioning the physically interesting fact that, as explicitly shown in [5-34], non-spherically symmetric rotating black holes in asymptotically flat spacetimes can support external stationary matter configurations which are made of (neutral or charged) massive scalar fields.
}

a e-mail: shaharhod@gmail.com black-hole spacetime have demonstrated explicitly that exponentially decaying quasi-bound state charged matter configurations, which are characterized by extremely long lifetimes, ${ }^{2}$ can be supported in the external spacetime region of the central charged black hole. These external quasi-bound field configurations are characterized by the physically motivated boundary condition of ingoing waves at the outer black-hole horizon and exponentially decaying (bounded) radial eigenfunctions at spatial infinity [37-42]. ${ }^{3}$

The main goal of the present paper is to explore the physical properties of these quasi-bound state charged matter configurations in the Reissner-Nordström black-hole spacetime. To this end, we shall analyze the Klein-Gordon wave equation for the charged massive scalar fields in the charged black-hole spacetime. Interestingly, as we shall explicitly show below, in the extremal $Q / M \rightarrow 1$ limit $^{4,5}$ of the central charged black hole, one can derive a remarkably compact analytical formula for the discrete frequency spectrum which characterizes the quasi-bound state resonances of the composed Reissner-Nordström-black-hole-chargedmassive-scalar-field configurations.

\footnotetext{
2 In particular, as shown in [35,36], the lifetimes of the external quasibound matter configurations can be very large on the scale $M$ set by the black-hole mass.

3 It is worth noting that the familiar quasinormal resonances (free oscillations) of the composed Reissner-Nordström-black-hole-chargedmassive-scalar-field system studied in [37-42] are characterized by the boundary condition of free outgoing waves at spatial infinity. On the other hand, as emphasized above, the quasi-bound state black-hole-field resonances that we shall study in the present paper are characterized by exponentially decaying (bounded) radial eigenfunctions at spatial infinity [see Eqs. (10) and (11) below] [35,36].

${ }^{4}$ We shall use natural units in which $G=c=\hbar=1$.

${ }^{5}$ Here $M$ and $Q$ are, respectively, the mass and electric charge of the central Reissner-Nordström black hole.
} 


\section{Description of the system}

We consider a scalar field $\Psi$ of mass $\mu$ and charge coupling constant $q^{6}$ which is linearly coupled to a ReissnerNordström black hole of mass $M$ and electric charged $Q .{ }^{7}$ The charged black-hole spacetime is described by the spherically symmetric metric [43]

$\mathrm{d} s^{2}=-f(r) \mathrm{d} t^{2}+\frac{1}{f(r)} \mathrm{d} r^{2}+r^{2}\left(\mathrm{~d} \theta^{2}+\sin ^{2} \theta \mathrm{d} \phi^{2}\right)$,

where

$f(r)=1-\frac{2 M}{r}+\frac{Q^{2}}{r^{2}}$.

The horizon radii which characterize the charged ReissnerNordström black-hole spacetime,

$r_{ \pm}=M \pm\left(M^{2}-Q^{2}\right)^{1 / 2}$

are determined by the zeros of the dimensionless metric function $f(r)$ [43].

The dynamics of the linearized charged massive scalar fields in the curved black-hole spacetime is governed by the familiar Klein-Gordon wave equation [37-42,44-48]

$\left[\left(\nabla^{v}-i q A^{v}\right)\left(\nabla_{v}-i q A_{v}\right)-\mu^{2}\right] \Psi=0$,

where $A_{v}=-\delta_{v}^{0} Q / r$ is the electromagnetic potential of the Reissner-Nordström black hole. Substituting into (4) the metric functions (1) and using the scalar field expansion ${ }^{8}$

$\Psi(t, r, \theta, \phi)=\int \sum_{\operatorname{lm}} e^{\mathrm{im} \phi} S_{\operatorname{lm}}(\theta) R_{\operatorname{lm}}(r ; \omega) e^{-i \omega t} \mathrm{~d} \omega$,

one finds the characteristic radial differential equation [4448]

$f(r) \frac{\mathrm{d}}{\mathrm{d} r}\left[r^{2} f(r) \frac{\mathrm{d} R}{\mathrm{~d} r}\right]+U R=0$

for the radial scalar eigenfunction $R(r)$, where ${ }^{9}$

$U=(\omega r-q Q)^{2}-f(r)\left[\mu^{2} r^{2}+l(l+1)\right]$.

${ }^{6}$ Note that $\mu$ and $q$ stand for $\mu / \hbar$ and $q / \hbar$, respectively. Hence, these physical parameters of the charged massive scalar fields have the dimensions of (length) ${ }^{-1}$.

${ }^{7}$ We shall assume $Q>0$ and $q>0$ for the composed black-hole-field system without loss of generality.

8 The integer parameters $m$ and $l \geq|m|$ are, respectively, the azimuthal harmonic index and the spherical harmonic index which characterize the scalar field mode. We shall henceforth omit these harmonic indices for brevity.

${ }^{9}$ Here $l(l+1)$ with $l=0,1,2, \ldots$ are the familiar discrete eigenvalues of the angular spherical harmonic functions $S_{\operatorname{lm}}(\theta)$.
We shall be interested in resonant modes of the charged massive scalar fields in the charged black-hole spacetime which are characterized by the physically motivated boundary condition $[2-4,8,12,14,35,35,36,49-75]$ :

$R\left(r \rightarrow r_{+}\right) \sim e^{-i\left(\omega-\omega_{c}\right) y}$

of purely ingoing waves (as measured by a comoving observer) at the outer horizon of the black hole, where

$\omega_{\mathrm{c}}=\frac{q Q}{r_{+}}$

is the critical (marginal) frequency for the superradiant scattering phenomenon of charged bosonic fields in the charged Reissner-Nordström black-hole spacetime [76], and the radial coordinate $y$ is defined by the relation $\mathrm{d} y / \mathrm{d} r=$ $1 / f(r) .{ }^{10}$ In addition, the quasi-bound state resonances of the linearized charged massive scalar fields that we shall analyze in the present paper are characterized by the asymptotic boundary condition $[2-4,8,12,14,35,35,36,49-75]$

$R(r \rightarrow \infty) \sim \frac{1}{r} e^{-\sqrt{\mu^{2}-\omega^{2}} r}$

of exponentially decaying radial eigenfunctions in the bounded regime

$\omega^{2}<\mu^{2}$

of small resonant frequencies.

The characteristic quasi-bound state resonances $\{\omega(\mu, q$, $l, M, Q ; n)\}^{11}$ of the charged massive scalar fields in the charged Reissner-Nordström black-hole spacetime are determined by the set of Eqs. (6), (8), and (10). Interestingly, as we shall explicitly show in the next section, the complex resonant spectrum which characterizes the quasi-bound state resonances of the composed Reissner-Nordström-black-holecharged-massive-scalar-field system can be determined analytically in the near-extremal $\left(r_{+}-r_{-}\right) / r_{+} \ll 1$ regime.

\section{The quasi-bound state resonances of the charged massive scalar fields in the charged Reissner-Nordström black-hole spacetime}

In the present section we shall study analytically the set of Eqs. (6), (8), and (10) which determine the quasi-bound state

\footnotetext{
${ }^{10}$ Note that the near-horizon limit $r \rightarrow r_{+}$corresponds to the asymptotic radial limit $y \rightarrow-\infty$.

11 Here the integer $n$ is the resonance parameter which characterizes the quasi-bound state resonances of the charged massive scalar fields [see Eq. (35) below].
} 
resonances of the charged massive scalar fields. In particular, below we shall derive a remarkably compact analytical formula for the complex resonant spectrum which characterizes the quasi-bound state massive scalar configurations in the charged Reissner-Nordström black-hole spacetime.

It proves useful to define the dimensionless physical parameters $[47,77,78]$

$$
\begin{aligned}
x & \equiv \frac{r-r_{+}}{r_{+}} ; \quad \tau \equiv \frac{r_{+}-r_{-}}{r_{+}} ; \quad k \equiv 2 \omega r_{+}-q Q ; \\
\varpi & \equiv \frac{\omega-\omega_{\mathrm{c}}}{2 \pi T_{\mathrm{BH}}}
\end{aligned}
$$

which characterize the composed Reissner-Nordström-blackhole-charged-massive-scalar-field system, where

$T_{\mathrm{BH}}=\frac{r_{+}-r_{-}}{4 \pi r_{+}^{2}}$

is the Bekenstein-Hawking temperature of the charged black hole. In addition, we shall use the dimensionless black-holefield parameter

$\beta^{2} \equiv(l+1 / 2)^{2}+\left(\mu r_{+}\right)^{2}-k^{2}$.

Below we shall assume $0<\beta \in \mathbb{R}^{12}$

Using the dimensionless parameters (12), one can express the radial differential Eq. (6) in the form [37,47]

$x(x+\tau) \frac{\mathrm{d}^{2} R}{\mathrm{~d} x^{2}}+(2 x+\tau) \frac{\mathrm{d} R}{\mathrm{~d} x}+U R=0$,

where the effective radial potential is given by

$$
\begin{aligned}
U= & U(x ; \mu, q, \omega, l, M, Q)=\frac{\left(\omega r_{+} x^{2}+k x+\varpi \tau / 2\right)^{2}}{x(x+\tau)} \\
& -l(l+1)-\left[\mu r_{+}(1+x)\right]^{2} .
\end{aligned}
$$

As shown in [37], for near-extremal black holes in the $\tau \ll 1$ regime, the characteristic differential equation (15) is amenable to an analytical treatment in the two asymptotic regions $x \ll 1$ and $x \gg \tau \times \max (1, \varpi)$ [13]. Interestingly, and most importantly for our analysis, by performing a standard matching procedure of the two analytical solutions [see Eqs. (18) and (26) below] in the overlapping radial region $\tau \times \max (1, \varpi) \ll x \ll 1,{ }^{13}$ one may determine the complex spectrum $\{\omega(\mu, q, l, M, Q ; n)\}$ of resonant frequencies which characterize the quasi-bound state configurations of the charged massive scalar fields in the charged ReissnerNordström black-hole spacetime.

\footnotetext{
12 It is worth mentioning that the case $i \beta \in \mathbb{R}$ was studied in [37].

13 Note that this matching procedure is valid in the low-temperature $\tau \ll 1$ regime of near-extremal black holes.
}

We shall first analyze the radial equation (15) in the nearhorizon region,

$x \ll 1$.

In this spatial region one may approximate the effective radial potential (16) by $U \rightarrow U_{\text {near }} \equiv(k x+\varpi \tau / 2)^{2} /[x(x+$ $\tau)]-l(l+1)-\left(\mu r_{+}\right)^{2}$. One can express the physically acceptable ${ }^{14}$ near-horizon solution of the radial differential Eq. (15) in the form $[13,37,79,80]$

$$
\begin{aligned}
R(x)= & x^{-i \frac{\varpi}{2}}\left(\frac{x}{\tau}+1\right)_{2}^{i \frac{\varpi}{2}-i k} F_{1}\left(\frac{1}{2}-\beta-i k,\right. \\
& \left.\frac{1}{2}+\beta-i k ; 1-i \varpi ;-x / \tau\right),
\end{aligned}
$$

where ${ }_{2} F_{1}(a, b ; c ; z)$ is the familiar hypergeometric function.

Using Eq. 15.3.7 of [80] one may express (18) in the form

$$
\begin{aligned}
R(x)= & x^{-i \frac{\varpi}{2}}\left(\frac{x}{\tau}+1\right)^{i \frac{\varpi}{2}-i k} \\
& \times\left[\frac{\Gamma(1-i \varpi) \Gamma(-2 \beta)}{\Gamma(1 / 2-\beta-i k) \Gamma(1 / 2-\beta+i k-i \varpi)}\left(\frac{x}{\tau}\right)^{-1 / 2-\beta+i k}\right. \\
& \times{ }_{2} F_{1}\left(\frac{1}{2}+\beta-i k, \frac{1}{2}+\beta-i k+i \varpi ; 1+2 \beta ;-\tau / x\right) \\
& +(\beta \rightarrow-\beta)],
\end{aligned}
$$

where the notation $(\beta \rightarrow-\beta)$ means "replace $\beta$ by $-\beta$ in the preceding term". Substituting into (19) the asymptotic expression (see Eq. 15.1.1 of [80])

${ }_{2} F_{1}(a, b ; c ; z) \rightarrow 1 \quad$ for $\frac{a b}{c} \cdot z \rightarrow 0$

one obtains [37]

$$
\begin{aligned}
R(x)= & \frac{\Gamma(1-i \varpi) \Gamma(-2 \beta) \tau^{1 / 2+\beta-i \varpi / 2}}{\Gamma(1 / 2-\beta-i k) \Gamma(1 / 2-\beta+i k-i \varpi)} x^{-\frac{1}{2}-\beta} \\
& +(\beta \rightarrow-\beta)
\end{aligned}
$$

in the intermediate spatial region

$\tau \times \max (1, \varpi) \ll x \ll 1$.

We shall next analyze the radial scalar equation (15) in the far-region

$x \gg \tau \times \max (1, \varpi)$.

In this spatial region one may approximate (15) by [37]

$x^{2} \frac{\mathrm{d}^{2} R}{\mathrm{~d} x^{2}}+2 x \frac{\mathrm{d} R}{\mathrm{~d} x}+U_{\mathrm{far}} R=0$,

${ }^{14}$ That is, the mathematical solution of the radial Eq. (15), which respects the physically motivated boundary condition (8) of purely ingoing waves at the outer black-hole horizon. 
where the effective far-region potential is given by $U \rightarrow$ $U_{\text {far }}=\left(\omega r_{+} x+k\right)^{2}-l(l+1)-\left[\mu r_{+}(1+x)\right]^{2}$. Defining the dimensionless variables

$\epsilon \equiv \sqrt{\mu^{2}-\omega^{2}} r_{+} ; \quad \kappa \equiv \frac{\omega k r_{+}-\left(\mu r_{+}\right)^{2}}{\epsilon}$,

one can express the far-region solution of radial differential Eq. (24) in the form $[13,37,79,80]$

$$
\begin{aligned}
R(x)= & N_{1} \times(2 \epsilon)^{\frac{1}{2}-\beta} x^{-\frac{1}{2}-\beta} e^{-\epsilon x} \\
& { }_{1} F_{1}\left(\frac{1}{2}-\beta-\kappa, 1-2 \beta, 2 \epsilon x\right)+N_{2} \times(\beta \rightarrow-\beta),
\end{aligned}
$$

where ${ }_{1} F_{1}(a, b, z)$ is the familiar confluent hypergeometric function and $\left\{N_{1}, N_{2}\right\}$ are normalization constants.

Substituting into (26) the asymptotic expression (see Eq. (13.1.2) of [80])

${ }_{1} F_{1}(a, b, z) \rightarrow 1$ for $\frac{a}{b} \cdot z \rightarrow 0$,

one obtains [37]

$R(x)=N_{1} \times(2 \epsilon)^{\frac{1}{2}-\beta} x^{-\frac{1}{2}-\beta}+N_{2} \times(\beta \rightarrow-\beta)$

in the intermediate spatial region

$\tau \times \max (1, \varpi) \ll x \ll 1$.

Matching the analytically derived radial solutions (21) and (28), which are both valid in the overlap region $\tau \times$ $\max (1, \varpi) \ll x \ll 1$, one finds the normalization constants ${ }^{15}[37]$

$$
\begin{aligned}
N_{1}(\beta)= & \frac{\Gamma(1-i \varpi) \Gamma(-2 \beta)}{\Gamma\left(\frac{1}{2}-\beta-i k\right) \Gamma\left(\frac{1}{2}-\beta+i k-i \varpi\right)} \tau^{\frac{1}{2}+\beta-i \frac{\varpi}{2}}(2 \epsilon)^{-\frac{1}{2}+\beta} \\
& \text { and } N_{2}(\beta)=N_{1}(-\beta)
\end{aligned}
$$

of the radial solution (26). In addition, using Eq. 13.5.1 of [80], one finds the asymptotic spatial behavior

$$
\begin{aligned}
R(x \rightarrow \infty) \rightarrow & {\left[N_{1} \times(2 \epsilon)^{\kappa} \frac{\Gamma(1-2 \beta)}{\Gamma\left(\frac{1}{2}-\beta+\kappa\right)} x^{-1+\kappa}(-1)^{-\frac{1}{2}+\beta+\kappa}\right.} \\
& \left.+N_{2} \times(\beta \rightarrow-\beta)\right] e^{-\epsilon x} \\
& +\left[N_{1} \times(2 \epsilon)^{-\kappa} \frac{\Gamma(1-2 \beta)}{\Gamma\left(\frac{1}{2}-\beta-\kappa\right)} x^{-1-\kappa}\right. \\
& \left.+N_{2} \times(\beta \rightarrow-\beta)\right] e^{\epsilon x}
\end{aligned}
$$

\footnotetext{
15 Note that the radial solutions (21) and (28) are characterized by the same functional dependence on the spatial coordinate $x$. This fact allows one to match these mathematical solutions in the overlap radial region $\tau \times \max (1, \varpi) \ll x \ll 1$.
}

for the radial eigenfunction (26). Remembering that the quasi-bound configurations of the charged massive scalar fields in the charged Reissner-Nordström black-hole spacetime are characterized by exponentially decaying radial eigenfunctions at spatial infinity [see Eqs. (10) and (11)], one arrives at the important conclusion that the coefficient of the exploding exponent $e^{\epsilon x}$ in the asymptotic spatial expression (31) should vanish:

$N_{1} \times(2 \epsilon)^{-\kappa} \frac{\Gamma(1-2 \beta)}{\Gamma\left(\frac{1}{2}-\beta-\kappa\right)} x^{-1-\kappa}+N_{2} \times(\beta \rightarrow-\beta)=0$.

Finally, substituting the normalization constants (30) into (32), one obtains the (rather cumbersome) resonance equation [37]

$$
\begin{aligned}
& {\left[\frac{\Gamma(2 \beta)}{\Gamma(-2 \beta)}\right]^{2} \frac{\Gamma\left(\frac{1}{2}-\beta-i k\right) \Gamma\left(\frac{1}{2}-\beta-\kappa\right) \Gamma\left(\frac{1}{2}-\beta+i k-i \varpi\right)}{\Gamma\left(\frac{1}{2}+\beta-i k\right) \Gamma\left(\frac{1}{2}+\beta-\kappa\right) \Gamma\left(\frac{1}{2}+\beta+i k-i \varpi\right)}} \\
& \quad=(2 \epsilon \tau)^{2 \beta}
\end{aligned}
$$

for the complex frequency spectrum $\{\omega(\mu, q, l, M, Q ; n)\}$, which characterizes the quasi-bound state resonances of the linearized charged massive scalar fields in the near-extremal charged Reissner-Nordström black-hole spacetime.

The assumption $0<\beta \in \mathbb{R}$ (see footnote 12) implies the strong inequality

$\delta \equiv(2 \epsilon \tau)^{2 \beta} \ll 1 \quad$ for $\tau \rightarrow 0$

in the low-temperature $\tau \rightarrow 0$ limit. In this regime of nearextremal $(\tau \rightarrow 0)$ black holes, the discrete resonant solutions of Eq. (33) can be expressed in the compact form

$\varpi_{n} \equiv k-i\left(n+\frac{1}{2}+\beta-\eta \cdot \delta\right) ; 0 \leq n \in \mathbb{Z}$.

The dimensionless constant $\eta$ in (35) can be determined by substituting the leading order expansion (see Eq. 6.1.34 of [80])

$\frac{1}{\Gamma\left(\frac{1}{2}+\beta+i k-i \varpi\right)}=(-1)^{n} n ! \eta \cdot \delta+O\left(\delta^{2}\right)$

into the 1.h.s. of the resonance equation (33). This yields

$\eta=\frac{\mathcal{F}}{(-1)^{n} n !}$,

where [see Eq. (33)]

$$
\begin{aligned}
\mathcal{F}= & {\left[\frac{\Gamma(-2 \beta)}{\Gamma(2 \beta)}\right]^{2} } \\
& \times \frac{\Gamma\left(\frac{1}{2}+\beta-i k\right) \Gamma\left(\frac{1}{2}+\beta-\kappa\right)}{\Gamma\left(\frac{1}{2}-\beta-i k\right) \Gamma\left(\frac{1}{2}-\beta-\kappa\right) \Gamma\left(\frac{1}{2}-\beta+i k-i \varpi\right)} .
\end{aligned}
$$




\section{Summary}

In summary, in this paper we have studied analytically the quasi-bound state resonances of charged massive scalar fields in the near-extremal charged Reissner-Nordström blackhole spacetime. These characteristic resonant modes of the composed black-hole-field system are characterized by the physically motivated boundary condition of purely ingoing waves at the black-hole horizon and asymptotically decaying (bounded) radial eigenfunctions at asymptotic spatial infinity.

Solving the Klein-Gordon wave equation for the linearized charged massive scalar fields in the near-extremal $\left(M T_{\mathrm{BH}} \rightarrow 0\right)$ charged black-hole spacetime, we have derived the compact analytical formula [see Eqs. (9), (12)-(14), (34), and (35) $]^{16}$

$$
\begin{aligned}
& \omega_{n}=\omega_{\mathrm{c}}+2 \pi T_{\mathrm{BH}} \\
& \cdot\left\{q Q-i\left[n+\frac{1}{2}+\sqrt{(l+1 / 2)^{2}+M^{2}\left(\mu^{2}-q^{2}\right)}\right]+O\left(M T_{\mathrm{BH}}, \delta\right)\right\} \\
& n=0,1,2, \ldots
\end{aligned}
$$

for the complex resonant spectrum which characterizes the quasi-bound state configurations of the composed ReissnerNordström-black-hole-charged-massive-scalar-field system.

Acknowledgements This research is supported by the Carmel Science Foundation. I thank Yael Oren, Arbel M. Ongo, Ayelet B. Lata, and Alona B. Tea for stimulating discussions.

Open Access This article is distributed under the terms of the Creative Commons Attribution 4.0 International License (http://creativecomm ons.org/licenses/by/4.0/), which permits unrestricted use, distribution, and reproduction in any medium, provided you give appropriate credit to the original author(s) and the source, provide a link to the Creative Commons license, and indicate if changes were made. Funded by SCOAP ${ }^{3}$.

\section{References}

1. A.E. Mayo, J.D. Bekenstein, Phys. Rev. D 54, 5059 (1996)

2. S. Hod, Phys. Lett. B 713, 505 (2012)

3. S. Hod, Phys. Lett. B 718, 1489 (2013). arXiv:1304.6474

4. S. Hod, Phys. Rev. D 91, 044047 (2015). arXiv:1504.00009

5. S. Hod, Phys. Rev. D 86, 104026 (2012). arXiv: 1211.3202

6. S. Hod, The Euro. Phys. Journal C 73, 2378 (2013). arXiv: 1311.5298

7. S. Hod, Phys. Rev. D 90, 024051 (2014). arXiv:1406.1179

8. S. Hod, Phys. Lett. B 739, 196 (2014). arXiv: 1411.2609

9. S. Hod, Class. Quantum Gravity 32, 134002 (2015). arXiv: 1607.00003

10. S. Hod, Phys. Lett. B 751, 177 (2015)

11. S. Hod, Class. Quantum Gravity 33, 114001 (2016)

12. S. Hod, Phys. Lett. B 758, 181 (2016). arXiv:1606.02306

\footnotetext{
${ }^{16}$ Here we have used the fact that $k=q Q+O\left(M T_{\mathrm{BH}}\right)$ in the nearextremal $T_{\mathrm{BH}} \rightarrow 0$ regime with $\omega=\omega_{\mathrm{c}}+O\left(T_{\mathrm{BH}}\right)$ [see Eqs. (9) and (12)].
}

13. S. Hod, O. Hod, Phys. Rev. D 81, 061502 (2010) (Rapid communication). arXiv:0910.0734

14. S. Hod, Phys. Lett. B 708, 320 (2012). arXiv: 1205.1872

15. S. Hod, J. High Energy Phys. 2017, 030 (2017). arXiv: 1612.00014

16. C.A.R. Herdeiro, E. Radu, Phys. Rev. Lett. 112, 221101 (2014)

17. C.L. Benone, L.C.B. Crispino, C. Herdeiro, E. Radu, Phys. Rev. D 90, 104024 (2014)

18. C.A.R. Herdeiro, E. Radu, Phys. Rev. D 89, 124018 (2014)

19. C.A.R. Herdeiro, E. Radu, Int. J. Mod. Phys. D 23, 1442014 (2014)

20. Y. Brihaye, C. Herdeiro, E. Radu, Phys. Lett. B 739, 1 (2014)

21. J.C. Degollado, C.A.R. Herdeiro, Phys. Rev. D 90, 065019 (2014)

22. C. Herdeiro, E. Radu, H. Rúnarsson, Phys. Lett. B 739, 302 (2014)

23. C. Herdeiro, E. Radu, Class. Quantum Gravity 32, 144001 (2015)

24. C.A.R. Herdeiro, E. Radu, Int. J. Mod. Phys. D 24, 1542014 (2015)

25. C.A.R. Herdeiro, E. Radu, Int. J. Mod. Phys. D 24, 1544022 (2015)

26. P.V.P. Cunha, C.A.R. Herdeiro, E. Radu, H.F. Rúnarsson, Phys. Rev. Lett. 115, 211102 (2015)

27. B. Kleihaus, J. Kunz, S. Yazadjiev, Phys. Lett. B 744, 406 (2015)

28. C.A.R. Herdeiro, E. Radu, H.F. Rúnarsson, Phys. Rev. D 92, $084059(2015)$

29. C. Herdeiro, J. Kunz, E. Radu, B. Subagyo, Phys. Lett. B 748, 30 (2015)

30. C.A.R. Herdeiro, E. Radu, H.F. Rúnarsson, Class. Quantum Gravity 33, 154001 (2016)

31. C. A. R. Herdeiro, E. Radu, H. F. Rúnarsson, arXiv:1604.06202

32. Y. Brihaye, C. Herdeiro, E. Radu, arXiv:1605.08901

33. Y. Ni, M. Zhou, A.C. Avendano, C. Bambi, C.A.R. Herdeiro, E. Radu, arXiv:1606.04654

34. M. Wang, arXiv:1606.00811

35. J.C. Degollado, C.A.R. Herdeiro, Gen. Rel. Grav. 45, 2483 (2013)

36. M.O.P. Sampaio, C. Herdeiro, M. Wang, Phys. Rev. D 90, 064004 (2014)

37. S. Hod, Phys. Lett. A 374, 2901 (2010). arXiv:1006.4439

38. S. Hod, Phys. Lett. B 710, 349 (2012). arXiv:1205.5087

39. S. Hod, Phys. Lett. B 747, 339 (2015). arXiv:1507.01943

40. S. Hod, Phys. Lett. B 761, 53 (2016). arXiv:1609.01297

41. R.A. Konoplya, A. Zhidenko, Phys. Rev. D 88, 024054 (2013)

42. M. Richartz, D. Giugno, Phys. Rev. D 90, 124011 (2014)

43. S. Chandrasekhar, The Mathematical Theory of Black Holes (Oxford University Press, New York, 1983)

44. S. Hod, T. Piran, Phys. Rev. D 58, 024017 (1998). arXiv:gr-qc/9712041

45. S. Hod, T. Piran, Phys. Rev. D 58, 024018 (1998). arXiv:gr-qc/9801001

46. S. Hod, T. Piran, Phys. Rev. D 58, 024019 (1998). arXiv:gr-qc/9801060

47. T. Hartman, W. Song, A. Strominger, JHEP 1003, 118 (2010)

48. S. Hod, Class. Quantum Gravity 23, L23 (2006). arXiv:gr-qc/0511047

49. T. Damour, N. Deruelle, R. Ruffini, Lett. Nuovo Cimento 15, 257 (1976)

50. S. Detweiler, Phys. Rev. D 22, 2323 (1980)

51. H. Furuhashi, Y. Nambu, Prog. Theor. Phys. 112, 983 (2004)

52. V. Cardoso, J.P.S. Lemos, Phys. Lett. B 621, 219 (2005)

53. V. Cardoso, S. Yoshida, JHEP 0507, 009 (2005)

54. S.R. Dolan, Phys. Rev. D 76, 084001 (2007)

55. S. Hod, O. Hod, (e-print). arXiv:0912.2761

56. H.R. Beyer, J. Math. Phys. 52, 102502 (2011)

57. Y.S. Myung, Phys. Rev. D 84, 024048 (2011)

58. J.P. Lee, JHEP 1201, 091 (2012)

59. J.P. Lee, Mod. Phys. Lett. A 27, 1250038 (2012)

60. S.R. Dolan, Phys. Rev. D 87, 124026 (2013)

61. H. Witek, V. Cardoso, A. Ishibashi, U. Sperhake, Phys. Rev. D 87, 043513 (2013)

62. V. Cardoso, Gen. Relat. Gravit. 45, 2079 (2013)

63. R. Li, Eur. Phys. J. C 73, 2274 (2013) 
64. S. J. Zhang, B. Wang, E. Abdalla, arXiv:1306.0932

65. H. Witek, arXiv:1307.1145

66. Y.S. Myung, Phys. Rev. D 88, 104017 (2013)

67. R. Li, Phys. Rev. D 88, 127901 (2013)

68. R. Brito, V. Cardoso, P. Pani, Phys. Rev. D 88, 023514 (2013)

69. H. Okawa, H. Witek, V. Cardoso, Phys. Rev. D 89, 104032 (2014)

70. B. Arderucio, arXiv: 1404.3421

71. H.M. Siahaan, Int. J. Mod. Phys. D 24, 1550102 (2015)

72. R. Brito, V. Cardoso, P. Pani, Lect. Notes Phys. 906, 1 (2015)

73. S. Hod, Phys. Lett. B 749, 167 (2015). arXiv:1510.05649
74. J.W. Gerow, A. Ritz, Phys. Rev. D 93, 044043 (2016)

75. Y. Huang, D. J. Liu, arXiv: 1606.08913

76. J.D. Bekenstein, Phys. Rev. D 7, 949 (1973)

77. S.A. Teukolsky, Phys. Rev. Lett. 29, 1114 (1972)

78. S.A. Teukolsky, Astrophys. J. 185, 635 (1973)

79. P.M. Morse, H. Feshbach, Methods of Theoretical Physics (McGraw-Hill, New York, 1953)

80. M. Abramowitz, I.A. Stegun, Handbook of Mathematical Functions (Dover Publications, New York, 1970) 